\title{
Coping with Type II diabetes: the patient's perspective
}

\author{
M. Koopmanschap* \\ Institute for Medical Technology Assessment, Erasmus University, Rotterdam, The Netherlands
}

\begin{abstract}
Aims/hypothesis. The Cost of Diabetes in Europe Type II study is the first coordinated attempt to assess the total costs of Type II (non-insulin-dependent) diabetes mellitus in Europe. Although gaining a clearer understanding of the economics of diabetes was the goal of the study, a secondary objective was to consider the impact of Type II diabetes from the patient's perspective.

Methods. The protocol included a health status questionnaire completed by the patient, the EQ-5D instrument, which assessed health-related quality of life by asking the patient to consider five dimensions: mobility, self-care, usual activities, pain and discomfort, as well as anxiety and depression.

Results. A total of 4189 patients in five of the eight countries completed the EQ-5D questionnaire for assessing the quality of life. Overall, patients with Type II diabetes reported scores between good and fair states of health, although the average score $(0.69)$ was lower than that of the similarly aged, healthy popula-
\end{abstract}

tion in the United Kingdom. However, these values were higher (0.76) in Type II diabetic patients without complications. The onset of either microvascular or macrovascular complications adversely affected quality of life ( 0.69 and 0.69 , respectively), and the presence of both types of complications further reduced the quality of life score to 0.59 . Treatment with insulin was also associated with a reduced quality of life (0.62). Multivariate analysis showed that the following factors, in order of importance, independently predict a poorer quality of life: gender, complications, treatment type, age, obesity and hyperglycaemia.

Conclusion/interpretation. The results showed that health-related quality of life is an important issue in Type II diabetes and this decreases with disease progression. The implication for policy-makers is that reducing or preventing the complications of diabetes is the key to improving patient quality of life. [Diabetologia (2002) 45:S18-S22]

Keywords CODE-2, quality of life, Type II diabetes.
Published online: 24 May 2002

(C) Springer-Verlag 2002

*M. Koopmanschap wrote on behalf of the CODE-2 Advisory Board

Corresponding author: Dr. M. Koopmanschap, Institute for Medical Technology Assessment, Erasmus University, PO Box 1738, 3000 DR Rotterdam, The Netherlands, E-mail: koopmanschap@bmg.eur.nl

Abbreviations: EQ-50, Euroqol 5 dimensions (questionnaire); CODE2, Cost of Diabetes in Europe Type 2; UKPDS, United Kingdom Prospective Diabetes Study; HRQoL, health-related quality of life.
Diabetes mellitus is a chronic metabolic disease that makes many demands on lifestyle, poses debilitating and life-threatening complications and has important implications for a patient's well-being and social life. The treatments for diabetes and its associated healthrisk factors are often highly complex and require considerable patient education and frequent medical monitoring.

Due to the expense of managing large numbers of patients with a host of associated morbidities, Type II diabetes represents a major burden to healthcare systems throughout the world [1]. A large proportion of the total costs for Type II diabetes are related to the 
management of the microvascular and macrovascular complications of the disease [2, 3, 4]. However, in addition to economic costs, there is often a substantial negative impact on an affected individual's wellbeing, family relationships and social life, leading to an overall reduction in health-related quality of life (HRQoL).

In general, patients with diabetes have a poorer quality of life than individuals without a chronic disease. The long-term complications of diabetes, such as retinopathy, neuropathy, heart disease and stroke, have a detrimental effect on quality of life. This is supported by the United Kingdom Prospective Diabetes Study (UKPDS) [5]. The burden of more than one complication often has an even greater impact on quality of life.

Measuring quality of life. Disease-specific instruments for the measurement of HRQoL have been used for a number of chronic conditions, including diabetes [6, 7]. These scales are useful for measuring the specific impact of the disease itself on patient well-being but they cannot be used to make comparisons with HRQoL in other diseases, with the general population or with patients with multiple morbidities. As an alternative, the use of general health status measurement tools for assessing HRQoL is becoming more frequent. These measurement tools focus on a patient's general well-being and ability to function in everyday life, and can be used to compare diseases and different populations.

In Europe, a frequently used tool for assessing HRQoL is the EQ-5D instrument, developed in 1987 by the EuroQoL research group [8, 9]. The EQ-5D scale provides an extensively validated tool for measuring HRQoL and was administered in the UKPDS trial [5]. This tool involves patient self-reporting of their health status in five dimensions: mobility, selfcare, usual activities, pain and discomfort, anxiety and depression.

Each dimension has three stages of limitation: no limitation, some limitation and extreme limitation. Processing a patient's self-completed report yields an HRQoL score between -0.59 and 1 , where 1 represents perfect health, 0 represents death and scores less than 0 represent health states perceived by the patient to be worse than death. The EQ-5D tool has the benefit that the scores obtained can be used as 'weighting values' in the construction of Quality Adjusted Life Years ( QALYs) used as the basis for cost-utility analysis [10].

\section{The CODE-2 study}

Obtaining comprehensive data on the total costs for patients with Type II diabetes in Europe was the purpose behind the design and implementation of the
Cost of Diabetes in Europe - Type II (CODE-2) study. Full details of the design of the CODE-2 study are presented elsewhere in this supplement [11]. To date, this is the first coordinated attempt to assess the total costs of patients with Type II diabetes in Europe. It has involved 7635 patients in eight European countries (Belgium, France, Germany, Italy, the Netherlands, Spain, Sweden and the UK). The objective of the study was to measure the total cost of patients with Type II diabetes in each country, providing data for international comparison. The study also had a number of secondary objectives, including consideration of factors from the patient perspective.

The CODE-2 study protocol included a patientcompleted questionnaire, which assessed the following items: HRQoL (EQ-5D); informal care, employment and retirement (to help calculate indirect costs); self-testing of blood glucose and glycosuria and satisfaction with treatment. In total, 4747 patients in five of the eight participating countries (Belgium, Italy, the Netherlands, Spain and Sweden) completed the patient questionnaire and the data was used for a subset analysis.

\section{Quality of Life in the CODE-2 study}

For the evaluation of HRQoL, the EQ-5D questionnaire was used. The questionnaire was completed by 4189 patients in five countries. The results of the analysis of HRQoL data in the CODE-2 study are presented as an average score for the total CODE- 2 population and as the variation in the HRQoL together with the status of complications and the type of diabetes treatment. To provide a context for these results, individual patient EQ-5D scores were correlated with the results of a self-rating health status item completed by the same patients. This provided average population EQ-5D values for diabetic patients reporting excellent, very good, good, fair and poor states of health $(0.95,0.92,0.83,0.64,0.27$ respectively). Univariate analysis of the HRQoL data used analysis of variance. All multivariate models and means were adjusted for stratification and country. The models used backward stepwise analysis of covariance to estimate the effect of each factor on HRQoL. A $p$ value of less than 0.05 was considered to be statistically significant.

Average Quality of Life in CODE-2 patients. The average HRQoL score in the CODE-2 patient cohort that completed the questionnaire is shown in Fig. 1. People with Type II diabetes in the CODE-2 study (mean age 66) reported an average HRQoL score of 0.69. This average score fell below the normal values of similarly aged populations in the United Kingdom ( 0.8 in the 55-64 age group and 0.78 in the 64-74 age group) [12]. However, all these scores fell within the interval between good and fair states of self-reported health 


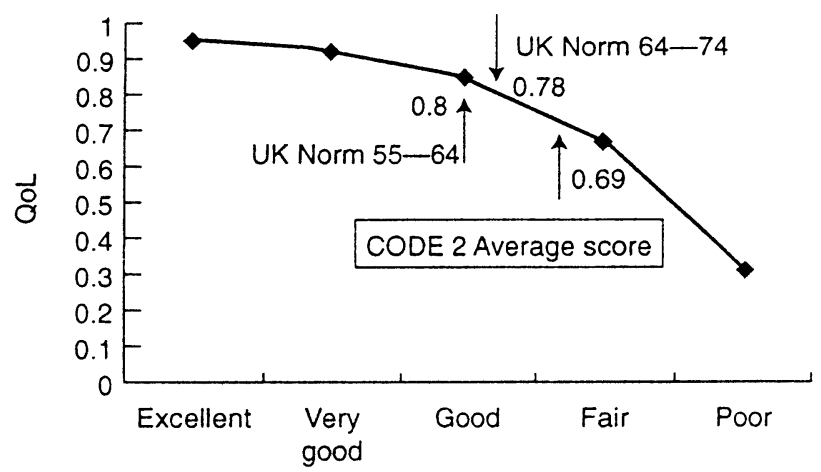

Fig. 1. Overall Quality of Life Score from patients in the CODE-2 population and in a general population of matched age, from the UK

and there were no differences between the average HRQoL scores for each of the participating countries.

Variation in Quality of Life by complication status. The HRQoL score was analysed as a function of the status of Type II diabetes complications, i.e. the presence of either microvascular or macrovascular complications or both in combination. The results show a clear correlation between HRQoL and the complication status (Fig. 2). In a multivariate model, the association between the presence of complications and HRQoL was statistically significant, even after adjusting for age, sex, treatment type, BMI, and $\mathrm{HbA}_{1 \mathrm{c}}$. Diabetic patients without complications had a HRQoL score of 0.76 . Microvascular or macrovascular complications only led to a reduction in HRQoL (scores 0.69 and 0.69 , respectively; $p=0.001$ ). While there was no difference in the HRQoL reported by patients with either microvascular or macrovascular complications, it was different from that reported by patients with no complications $(p=0.001)$. However, both types of complications resulted in a further reduction in the average HRQoL (0.59), compared with that for patients with any complication taken alone or for patients without complications $(p=0.001)$. This relation was present after adjusting for country and stratification.

Variation in quality of life by treatment type. When variation in HRQoL was considered according to treatment type, there was no difference in patients requiring treatment with oral antidiabetic drugs, compared with those maintained on diet and exercise alone (0.71 vs 0.71 , respectively) (Fig. 3 ). However,

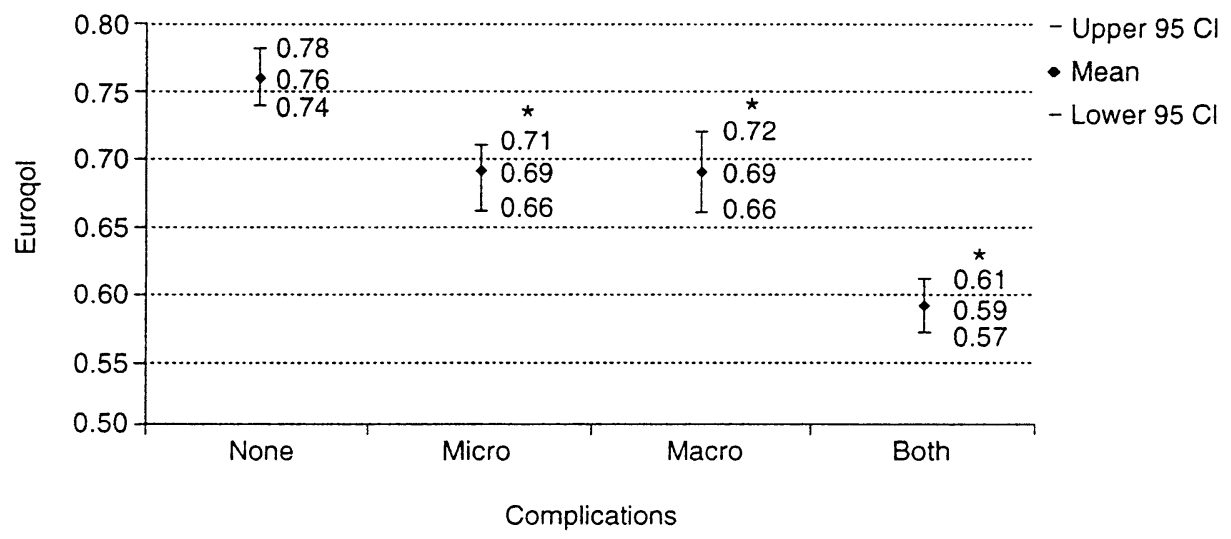

Fig. 2. Health-related quality of life (HRQoL) analysed by function of microvascular, macrovascular complications or both. ${ }^{*} p<0.001$ when compared to no complications

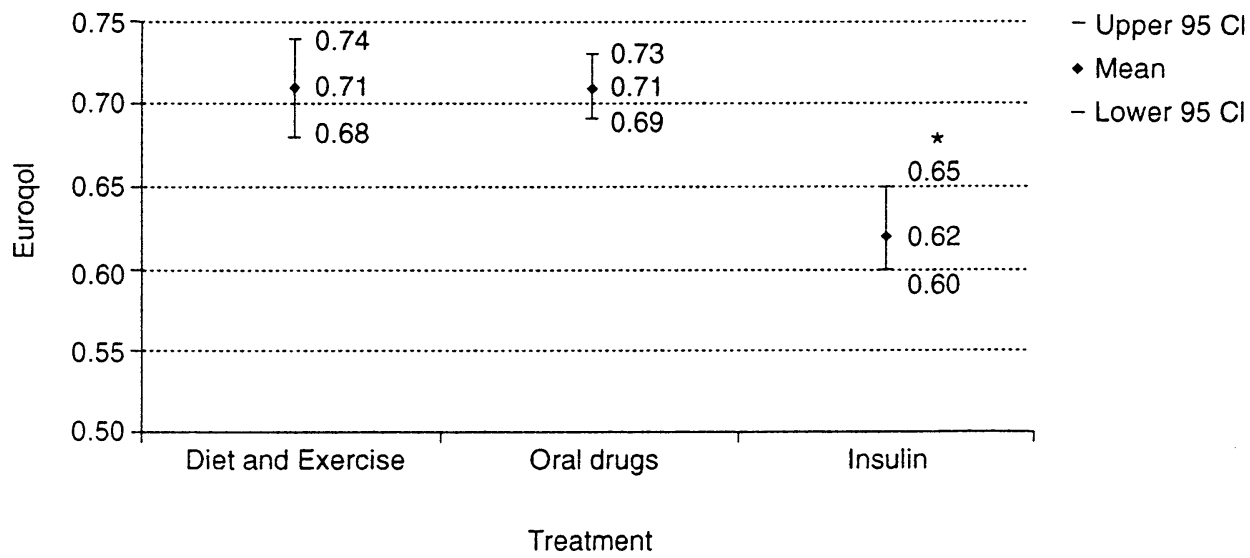

Fig. 3. Variations in HRQoL depending on the type of therapy received. ${ }^{*} p<0.001$ 
in patients requiring insulin for glycaemic control HRQoL was reduced to a mean value of 0.62 ( $p=$ 0.001 ). In a multivariate model, treatment type was a predictor of quality of life after adjusting for other variables, such as age, sex and any complications.

Other multivariate analysis results. Other independent predictors of poor quality of life were age, sex (female), BMI (obesity), and $\mathrm{HbA}_{1 \mathrm{c}}$ (poor glycaemic control).

\section{Discussion}

HRQoL is one of the main measures used to evaluate the influence of the management of chronic disorders on health but it is rarely measured in burden of illness studies. The general definition of health is "a state of complete physical, mental and social well-being, and not merely the absence of disease or infirmity" [13]. More specifically, HRQoL "refers to the physical, psychological and social domains of health that are influenced by a person's experiences, beliefs, expectations and perceptions" [14]. Although Type II diabetes is often incorrectly perceived as a relatively mild disease in comparison with Type I diabetes, the consequences for the patient's HRQoL are likely to be considerable, consisting of [15]: psychological effects associated with reduced general well-being; family life effects, arising from a lack of acceptance and support from family members; social effects, such as reduced social function due to feelings of isolation and withdrawal

Studies indicate that diabetic patients generally have conditions such as depression and anxiety more often than the general population of a similar age [16]. Fatigue, loss of enjoyment and reduced leisure pursuits also seem to correlate positively with the presence of Type II diabetes [17] as do feelings of restriction when complying with treatment strategies and self-monitoring requirements. In particular, the demand for increased physical activity, as part of a disease management regimen, can be highly stressful to obese patients.

Type II diabetic patients frequently report a reduction of family function and social life. There are indications that maintenance of social function can prevent psychological disorders such as depression [18]. Furthermore, a high degree of family acceptance and support has been shown to correlate with adherence to a treatment regimen and hence the ability to achieve and maintain good glycaemic control [19].

The long-term complications of Type II diabetes also can have pronounced effects on the patient's HRQoL, both at an early stage due to anxiety over future problems and later when the complications manifest themselves. Macrovascular abnormalities give rise to cardiovascular complications associated with high morbidity and mortality rates, such as angina pectoris, myocardial infarction and stroke. Microvascular changes can result in visual impairment as a consequence of diabetic retinopathy, in limb amputation in patients with diabetic neuropathy and in dialysis and kidney transplantation in patients with diabetic nephropathy. Each of these complications can diminish social function and independence. The prevalence of complications is high in people with Type II diabetes, for example, $50-75 \%$ of patients will be affected by mild retinopathy during their lifetime, with legal or total blindness being experienced by $19-20 \%$ of Type II diabetic patients [20]. A study based in England showed that over $40 \%$ of patients with Type II diabetes had neuropathy [21]. Complications can therefore have a considerable impact on the quality of life of a large proportion of Type II diabetic patients.

The results of the CODE-2 study have confirm that HRQoL is an important issue in Type II diabetes. Patients with Type II diabetes have moderately lower HRQoL scores than the general population of similar age. Furthermore, HRQoL deteriorates with disease progression, treatment progression and the development of complications. In a small patient cohort, progression from oral agents to insulin was associated with an increase in HRQoL, which the authors attributed to sustained, reduction in $\mathrm{HbA}_{1 \mathrm{c}}$ [22]. In contrast to this, the CODE-2 study shows that progression to insulin treatment is independently associated with a reduction in quality of life as is poor glycaemic control and a BMI of more than 27.

In conclusion, the implication for policy makers is that an avoidance of insulin therapy and the reduction or prevention of complications is the key to improving patients' HRQoL [4].

\section{References}

1. King H, Aubert RE, Herman WH (1998) Global burden of diabetes, 1995-2025. Diabetes Care 21:1414-1431

2. Huse DM, Oster G, Killen AR, Lacey MJ, Colditz GA (1989) The economic costs of non-insulin-dependent diabetes mellitus. JAMA 262:2708-2713

3. Brown JB, Pedula KL, Bakst AW (1999) The progressive cost of complications in Type 2 diabetes mellitus. Arch Intern Med 159:1873-1880

4. Williams R, Van Gaal L, Lucioni C (2002) Assessing the impact of complications on the costs of Type 2 diabetes. Diabetologia supplement (this issue)

5. UK Prospective Diabetes Study group (1999) Quality of life in Type 2 diabetes patients is affected by complications but not by intensive policies to improve blood glucose or blood pressure control. Diabetes Care 22:1125-1136

6. Shen W, Kotsanos JG, Huster WJ, Mathias SD, Andrejasich CM, Patrick DL (1999) Development and validation of the Diabetes Quality of Life Clinical Trial Questionnaire. Med Care 37:AS45-AS66

7. Hermann BP, Vickrey B, Hays RD et al. (1996) A comparison of health-related quality of life in patients with epilepsy, diabetes and multiple sclerosis. Epilepsy Res 25:113118 
8. Brooks B with the EuroQoL Group (1996) EuroQoL: The current state of play. Health Policy 37:52-72

9. EuroQoL User Guide (1996) Euroqol Business Management Group, Rotterdam

10. Drummond MF, O'Brien B, Stoddart GL, Torrance GW (1997) Methods for the Economic Evaluation of Healthcare Programmes, 2nd edn. Oxford University Press, Oxford

11. Jonsson B (2000) Revealing the cost of Type 2 diabetes in Europe. Diabetologia supplement (this issue)

12. Kind P, Dolan P, Gudex C, Williams A (1998) Variations in population health status: results from a United Kingdom national questionnaire survey. BMJ 316:736-741

13. World Health Organisation (1948) World Health Organisation constitution. In: Basic Documents. World Health Organisation, Geneva New Eng J Med 334(13):835-840

14. Testa MA, Simonson DC (1996) Assessment of quality-oflife outcomes. N Engl J Med 334:835-840

15. Assal JP (1995) Cost-effectiveness of diabetes education. Pharmacoeconomics 8:68-71

16. Jacobson AM (1996) The psychological care of patients with insulin dependent diabetes mellitus. $\mathrm{N}$ Engl J Med 334:1249-1253
17. Hörnquist JO, Wikby A, Stenström U, Andersson PO, Åkerlind I (1995) Type II diabetes and quality of life. Pharmacoeconomics 8:12-16

18. Littlefield C, Rodin GM, Murray MA, Craven JL (1990) Influence of functional impairment and social support on depressive symptoms in persons with diabetes. Health Psychol 9:737-749

19. Cox DJ, Gonder-Frederick L (1992) Major developments in behavioural diabetes research. J Consult Clin Psychol 60:628-638

20. Cunha-Vaz J (1998) Lowering the risk of visual impairment and blindness. Diabet Med 15:S47-S50

21. Kumar S, Ashe HA, Parnell LN et al. (1994) The prevalence of foot ulceration and its correlates in Type 2 diabetic patients: a population-based study. Diabet Med 11:480484

22. Pibernik-Okanoviæ M, Szabo S, Metelko Z (1998) Quality of life following a change in therapy for diabetes mellitus. Pharmacoeconomics 14:201-207 